\title{
Beck and back: A paradigm change in coronary sinus interventions-pulsatile stretch on intact coronary venous endothelium
}

Guenter Weigel, MD, ${ }^{a}$ llinka Kajgana, $\mathrm{PhD},{ }^{\mathrm{b}}$ Helga Bergmeister, MD, DVM, ${ }^{\mathrm{c}}$ Gunther Riedl, MD, ${ }^{\mathrm{a}}$ Helmut-Dietmar Glogar, MD, ${ }^{d}$ Mariann Gyöngyösi, MD, ${ }^{d}$ Stefan Blasnig, ${ }^{f}$ Georg Heinze, PhD, ${ }^{\mathrm{e}}$ and Werner Mohl, MD, PhD ${ }^{\mathrm{a}}$

Supplemental material is available online.
From the Departments of Cardiothoracic Surgery $^{\mathrm{a}}$ and Internal Medicine 2, Division of Cardiology, ${ }^{\mathrm{d}}$ Institute of Biomedical Research, ${ }^{\mathrm{c}}$ and Core Unit for Medical Statistics and Informatics, Section of Clinical Biometrics, ${ }^{\text {e }}$ Medical University of Vienna; and TU BioMed, University of Technology, ${ }^{\mathrm{b}}$ Vienna, Austria; and Carinthia University of Applied Sciences, Medical Information Technology, Klagenfurt, Austria. ${ }^{f}$

This work was supported by the "Fonds zur Foerderung der wissenschaftlichen Forschung P 13274-Med," the "Society of Coronary Sinus Interventions," the Federal Ministry of Traffic, Innovation and Technology (BMVIT) GZ609.637/0002-III/I2/ 2004, the Ludwig Boltzmann Institute for Cardiosurgical Research, and the Austrian Research Center Seibersdorf.

Werner Mohl reports patents on PICSO.

Received for publication July 22, 2006; revisions received Nov 20, 2006; accepted for publication Dec 28, 2006.

Address for reprints: Werner Mohl, MD, $\mathrm{PhD}$, Medical University of Vienna, Department of Cardiothoracic Surgery, Waehringer Guertel 18-20, 1090 Vienna, Austria (E-mail: werner.mohl@meduniwien. ac.at).

J Thorac Cardiovasc Surg 2007;133:1581-7 $0022-5223 / \$ 32.00$

Copyright (c) 2007 by The American Association for Thoracic Surgery

doi:10.1016/j.jtcvs.2006.12.044
Objectives: Strategies to recover myocardium in therapeutically unresponsive patients are again under scrutiny, including techniques developed in the pioneering days of cardiothoracic surgery such as retroperfusion via the coronary sinus-the Beck procedure. An underestimated aspect of retroperfusion is the formation of new vessels. This early observation of neoangiogenesis may be an important mechanism in observed benefits. We hypothesized that periodic pressure elevation in coronary veins induces an analogy to shear stress angiogenic pulses by activating venous endothelium. Pulsatile stretch on venous endothelium can be achieved easily by a pressure-controlled intermittent balloon blockade of the coronary sinus outflow.

Methods: Three hours of myocardial ischemia was induced in 12 pigs. Pressurecontrolled intermittent coronary sinus occlusion was applied in 6 animals 15 minutes after occlusion of the left anterior descending coronary artery. Postmortem myocardial specimens were taken, and heme oxygenase-1, vascular endothelial growth factor gene expression, and hypoxia-induced factor activity were measured.

Results: As compared with controls, treated animals released an angiogenic pulse by a 4-fold increase of heme oxygenase-1 gene expression in the infarct area $(P<$ $.001)$, together with a 2.5 -fold enhanced transcription of vascular endothelial growth factor in the infarct $(P<.006)$, border $(P<.002)$, and remote $(P<.02)$ areas, whereas hypoxia-induced factor activity was similar in both groups. A significant correlation $(P<.01)$ of the achieved coronary sinus pressure elevation and gene expression was found.

Conclusions: Mechanotransduction of pulsatile stretch on coronary venous endothelium by pressure-controlled intermittent coronary sinus occlusion induces heme oxygenase-1 and vascular endothelial growth factor gene expression, leaving the ischemic pathway of the hypoxia-induced factor activity unchanged. This cascade of molecular events closes the argument gap to historical reports of the Beck procedure on revascularization and myocardial salvage.

$\mathrm{T}$ oday, in the advent of percutaneous techniques, cardiac tissue regeneration to treat therapeutically unresponsive patients remains one of the important fields in cardiac research. Many of the methods developed by our surgical forefathers are again under scrutiny because alternative methods are increasingly used by cardiologists to induce regeneration and recovery of the failing heart. Although many of these pioneering techniques were abandoned because of the fast track of early technological development in cardiothoracic surgery in days past, new interest on the repair mechanism, stem cell research, cell transplantation, and methods to accomplish improved homing, focus interest on the historical perspective. ${ }^{1,2}$ Recently Kanamori and associates ${ }^{3}$ reported on a hybrid surgical angiogen- 


\author{
Abbreviations and Acronyms \\ $\beta 2-\mathrm{M}=\beta 2$-microglobulin \\ HIF = hypoxia-induced factor \\ HO heme oxygenase \\ LAD $=$ left anterior descending coronary artery \\ $\mathrm{PICSO}=$ pressure-controlled intermittent coronary \\ sinus occlusion \\ VEGF $=$ vascular endothelial growth factor
}

esis using cardio-omentopexy, a method introduced as early as 1936 by O'Shaughnessy. ${ }^{4}$ One of the pioneers in the use of cardio-omentopexy was Claude Beck, who also developed a clinically applicable surgical retroperfusion technique using the coronary sinus route. Although experimental and clinical results showed considerable improvements in many of the individuals treated, the effects of this procedure on the reversal of myocardial ischemia may have been misinterpreted and ever since have been the subject of controversial debate.

There is no doubt, considering everyday surgical practice, that reversal of coronary flow can protect the globally arrested myocardium and even start the heart beating during retroperfusion of warm blood at the end of aortic clamping. However, the question remains whether retroperfusion of arterial blood in the regionally ischemic beating heart is the cause of improvements observed.

The salvage potential of different retroperfusion techniques originating from Beck's idea have been established in a meta-analysis showing an inverse relationship between achieved coronary sinus pressure (duration and elevation of the coronary venous pressure during retroperfusion) and infarct size. This obvious relationship between the hemodynamic force of periodic elevation of coronary venous pressure on jeopardized myocardium and salvage has never been appreciated, because the scientific mainstream associated beneficial effects of retroperfusion techniques to the delivery of oxygenated blood. ${ }^{5}$ In this context, it is noteworthy that the fact that myocardial salvage less likely occurs in Krogh-like diffusion cylinders around major retroperfused cardiac veins, but rather in border zones of deprived myocardium, has never been questioned.

An important implication to solve this question is an observation made in a historical report by Hahn and Kim, ${ }^{6}$ studying the Beck procedure, on long-term histologic changes of ischemic myocardium describing "many vascular channels distal to the occluded artery." This observation is of special interest and can be interpreted as neoangiogenesis. Concerning this obvious form of revascularization and hyperplasia of veins subjected to permanent reversal of flow, the authors commented "that pulsatile pressure might be the origin of these changes," but that their historical study was unable to answer this question. ${ }^{6}$
An integral component of the healing process after myocardial infarction is the development of neoangiogenesis in the myocardial infarct zone. Under normal circumstances, the contribution of neoangiogenesis to the infarct-bed capillary network is insufficient to keep pace with the tissue growth required for contractile compensation. Several methods have been postulated to induce neoangiogenesis to recover the structural and functional integrity of jeopardized myocardium, but they remain a matter of debate. ${ }^{7}$

Evidently, Hahn and Kim's observation ${ }^{6}$ corresponds to a form of neovascularization. It can be speculated whether pulsatile pressure and/or other factors such as cytokine release might be involved.

Alternatively, the induction of genomic reprogramming for cell survival, including cytoprotective genes via environmental changes such as mechanotransduction and activation of endothelium, are well established. ${ }^{89}$ Since it is suggested that mechanical factors play a major role in coronary angiogenesis, ${ }^{10-12}$ a biomechanical intervention should affect myocardial gene expression. It has been shown that shear stress on the endothelium during ischemia induces signaling pathways leading to the formation of collaterals via pressure and flow gradients within the microcirculation and consequently to increased shear stress. Our objective in this study was to use this otherwise not influenceable natural mechanism intentionally with periodic venous pressure elevation (pressure-controlled intermittent coronary sinus occlusion-PICSO) in a pig model and to link it to known effects of retroperfusion like myocardial salvage and Hahn and Kim's histologic observation of an induction of vessel growth.

To study the molecular cascade induced by mechanical force on endothelium, we focus on two functionally interrelated, proangiogenic genes in porcine myocardium: heme oxygenase (HO)-1, a molecule also known to be involved in vasodilation, and vascular endothelial growth factor (VEGF). We anticipate that the microcirculatory stretch induced by PICSO is associated with the upregulation of HO-1 and VEGF mRNA, two markers expressed by the activated endothelium, whereas the ischemic pathway via hypoxia-induced factor (HIF) activity should remain unchanged. The purpose of this study was to close the gap between the experience in coronary sinus retroperfusion of the past and our hypothesis that pulsatile pressure in the coronary venous circulation may play a role in myocardial salvage and neoangiogenesis.

\section{Materials and Methods}

(See additional text online.)*

The animals were given systemic anticoagulation with a heparin sulfate intravenous bolus (1000 IU) before preparation of the great neck vessels. The heart was exposed through an incision in the fifth left intercostal space, part of the fifth rib was removed, and the pericardium was cut open and sutured to the chest wall. The 
heart was then suspended in a pericardial cradle. The left anterior descending artery (LAD) and the circumflex artery were carefully dissected free of their venae comitans with the help of local immobilization normally used in beating heart surgery. The LAD was then encircled with a vascular snare distal to the major diagonal branches. Before catheterization of the coronary sinus, a heparin sulfate bolus (20,000 IU, intravenous) was administered. A double-lumen 7F catheter with a curved tip (Contract Medical International $\mathrm{GmbH}$, Dresden, Germany) was inserted from the left internal jugular vein and placed in a stable position with the balloon approximately $25 \mathrm{~mm}$ inside the coronary sinus. Then the hemiazygos vein draining the coronary sinus was occluded. The correct balloon location and complete occlusion of the coronary sinus were confirmed radiographically during one occlusion cycle. The coronary sinus pressure was monitored and recorded continuously through the central lumen of the catheter. The stable position of the balloon during occlusion and release was maintained through monitoring the balloon pressure. During the entire experiment, all measurements were recorded on a computerized data acquisition system for biological signals.

\section{Study Protocol}

All animals received human care in compliance with the "Principles of Laboratory Animal Care," formulated by the Institute of Laboratory Animal Resources and the "Guide for the Use of Laboratory Animals," prepared by the National Academy of Sciences and published by the National Institutes of Health (Pub No. 86-23, revised 1985). A series of 12 pigs (6 PICSO treated vs 6 controls) was subjected to 3 hours of ischemia. The previously placed vascular snare around the left anterior descending artery (LAD) was occluded, with a 3-hour period of acute anterior wall ischemia. Six pigs received no further intervention (control group). In the study group, PICSO intervention was started 15 minutes after LAD occlusion and continued throughout the entire experiment. The pump cycles ranged from 5 seconds of inflation $/ 3$ seconds of deflation to 10 seconds of inflation $/ 5$ seconds of deflation. In a subset of animals (4 PICSO-treated/2 controls), global wall motion was determined in regular intervals with epicardial 2-dimensional echocardiography. Hemodynamic data were recorded throughout the experiment.

\section{PICSO}

A coronary sinus pressure-controlled feedback system was developed to produce intermittent coronary sinus occlusion and has been reported elsewhere (Austrian Research Center, Seibersdorf, Austria). Continuous measurements of the coronary sinus pressure and an effective feedback control allowed a physiologic pressure increase without reactive and permanent coronary inflow impedance. ${ }^{13}$

(See additional text online. $)^{\dagger}$

\section{Internal End-diastolic and End-systolic Diameters}

Two-dimensional echocardiograms were recorded on a cine loop so that at least 2 or 3 measurements of the cavity and myocardium could be taken in cross sections and long-axis views. Left ventricular end-systolic and end-diastolic volumes were calculated from 2-dimensional echocardiograms by the area-length method by the following formula: $\mathrm{V}=(8 / 3 \pi) \cdot\left(\mathrm{A}_{\max }{ }^{2} / \mathrm{IL}_{\max }\right)$, where $\mathrm{V}$ presents the volume of the ventricle, $\mathrm{A}_{\max }$ is the maximal cross-sectional area of the ventricle at the midpapillary muscle level, and $\mathrm{L}_{\max }$ expresses the maximal length of the ventricle between the mitral valve and apex. End-diastolic (EDV) and end-systolic volumes (ESV) were calculated by measuring the corresponding end-diastolic and end-systolic $A_{\max }$ and $L_{\max } \cdot{ }^{E 1}$ Ejection fraction was calculated as (EDV - ESV)/EDV and expressed as percentage. Heart rate was monitored continuously. The recordings were evaluated by an observer blinded to the treatment group.

\section{RNA Extraction and cDNA Synthesis}

At the end of each experiment, the animal was put to death and the heart was removed quickly. After being rinsed with a cold $0.9 \%$ sodium chloride solution, the heart was dipped in liquid nitrogen for approximately 20 seconds. For the RNA extraction small pieces of tissue, representing the infarct area, the peri-infarct border area, and the remote noninfarcted myocardium, were cut from frozen slices of infarcted pig hearts. These small pieces of pig heart tissue (approximately $150 \mathrm{mg}$ [wet weight]) were homogenized in a tissue grinder, total RNA was extracted with TriReagent (Molecular Research Center, Cincinnati, Ohio) according to the manufacturer's protocol, and $1 \mu \mathrm{g}$ of the isolated total RNA was reverse transcribed.

\section{Primers and Taq Man Probes}

Primers and FAM-labeled Taq Man probes for HO-1 and VEGF were designed according to GenBank: accession numbers X60677 and AF461807, respectively. Primers were designed to exclude the amplification of genomic DNA. Target transcripts were normalized to the endogenous housekeeping transcript $\beta 2$-microglobulin $(\beta 2-\mathrm{M})$. Primers and FAM-labeled Taq Man probe were designed according to GenBank: accession number L13854.

(See additional text online. $)^{\ddagger}$

\section{Real-time Quantitative Reverse Transcriptase-Polymerase Chain Reaction}

Fifty nanograms of cDNA was amplified in a Gene Amp 5700 Sequence Detection System (Applied Biosystems, Foster City, Calif), and polymerase chain reaction results were analyzed with the Sequence detector 1.6 program (Perkin-Elmer, Inc, Waltham, Mass). The amount of target was normalized to the endogenous reference $\beta 2-\mathrm{M}$. Analyses were performed in triplicate.

\section{Assay of HIF-1 $\alpha$ Activity: Electrophoretic Mobility Shift Assay}

Myocardial specimens (200 mg) from remote, border, and infarcted areas were washed twice with ice-cold phosphate-buffered saline containing $1 \mu \mathrm{g} / \mathrm{mL}$ leupeptin (Valeant Pharmaceuticals International, Costa Mesa, Calif), $1 \mu \mathrm{g} / \mathrm{mL}$ aprotinin (ICN Pharmaceuticals), and $0.5 \mathrm{mmol} / \mathrm{L}$ phenylmethylsulfonylfluoride (Sigma Chemical Company, St Louis, Mo). Tissue samples were transferred into a tissue grinder and homogenized in $5 \times$ volume cold extraction buffer consisting of $20 \mathrm{mmol} / \mathrm{L}$ HEPES ( $\mathrm{pH} 7.9$ ), $0.45 \mathrm{~mol} / \mathrm{L} \mathrm{NaCl}, 1 \mathrm{mmol} / \mathrm{L}$ ethylenediaminetetraacetic acid, 1 $\mu \mathrm{g} / \mathrm{mL}$ leupeptin, $1 \mu \mathrm{g} / \mathrm{mL}$ aprotinin, and $0.5 \mathrm{mmol} / \mathrm{L}$ phenylmethylsulfonylfluoride (Sigma). Thereafter, the homogenate was transferred to an Eppendorf tube and incubated on ice on a rocking 
platform for 10 minutes. The samples were then sonicated on ice in a bath-type sonicator $(2 \times 60$ seconds $)$, and extracted material was recovered as the supernatant after centrifugation $(12,500 \mathrm{~g}$ for 10 minutes at $4^{\circ} \mathrm{C}$ ). The supernatants were diluted with equal volumes of an ice-cold buffer consisting of $20 \mathrm{mmol} / \mathrm{L}$ HEPES, $0.1 \mathrm{~mol} / \mathrm{L} \mathrm{KCl}, 0.2 \mathrm{mmol} / \mathrm{L}$ ethylenediaminetetraacetic acid, $20 \%$ (v/v) glycerol, and $0.5 \mathrm{mmol} / \mathrm{L}$ dithiothreitol and immediately frozen in liquid nitrogen. Protein content of the recovered extracts was determined against solutions with increasing concentrations of bovine serum albumin standard using a modified Bradford protein assay (Bio-Rad Laboratories, Hercules, Calif). The extracts retrieved from myocardial specimens were subjected to the DNA binding reaction with the HIF- $1 \alpha$ specific oligonucleotide probe.

(See additional text online.) ${ }^{\S}$

\section{Statistical Analysis}

Data are given as mean \pm standard deviation except where indicated. The effect of coronary sinus occlusion on gene expression was analyzed by the Student 2-sample $t$ test on log-base-2 transformed data. The course of hemodynamic parameters for each pig during LAD occlusion was summarized by computing the individual average value as summary measure. ${ }^{13}$ Groups were then compared by analysis of covariance, adjusting for individual baseline values. ${ }^{14}$ Similarly, functional parameters were compared between groups by analysis of covariance of the individual average values, adjusting for individual baseline values. Changes from baseline values within the groups were assessed by paired $t$ test. Correlation analysis was performed by computing the Pearson linear correlation coefficient.

\section{Results}

\section{Hemodynamics}

Intermittent coronary sinus occlusion resulted in a gradual increase of the coronary venous pressure, reaching a plateau after a few seconds. After release of the outflow impedance, pressures returned to control values almost immediately. (See additional text online. $)^{\mathrm{T}}$

Comparison between groups showed that PICSO led to a strong increase of the systolic coronary sinus pressure (57 \pm $14.2 \mathrm{~mm} \mathrm{Hg}$ ), whereas the systolic coronary sinus pressure in the control group during LAD ligation was $8 \pm 2.35 \mathrm{~mm}$ $\mathrm{Hg}$. Comparison of the arterial pressure after LAD ligation showed no difference between groups. To avoid negative side effects of coronary outflow impedance, we timed coronary sinus occlusion according to systolic venous pressure increase. This is in accordance with the hypothesis that intermittent coronary sinus occlusion has to be adjusted to individual pressures to optimize its effectiveness and avoid prolonged periods of an increase of coronary inflow impedance, which is a severe counteractive and unwanted side effect of coronary venous pressure increase. Normally, prolonged and fixed timings of coronary sinus occlusion with subsequent increments in venous outflow impedance lead to changes in arterial inflow. It is known that prolonged coronary sinus occlusion induces arterial hypotension as well as bradycardia owing to excessive impedance increments for arterial inflow, effects that were not found in this set of experiments. The implementation of an automatic feedback control of coronary venous pressure elevations restricted these side effects of coronary sinus reflexes and improved regional perfusion, which even resulted in a tendency toward higher pressures and stable hemodynamics in the PICSO group.

Measurements of the ejection fraction as estimate of global myocardial function are depicted in Figure E1. Ventricular dimensions and volumes during the time course of the experiment are depicted in Figures E2 to E5. Although the number of animals allows only a limited interpretation, the data suggest that global contraction was preserved in PICSO-treated animals as compared with controls (although function declined in both groups, a statistically significant decrease could be observed only in the control group).

\section{Patterns of Gene Expression and Tissue Distribution of Transcripts}

We tested the hypothesis that VEGF, an endothelial cell mitogen and a major regulator of angiogenesis, is upregulated in response to intermittent occlusion of the coronary sinus. In PICSO-treated animals, a statistically significant upregulation of VEGF expression was observed (Figure E6). In all areas investigated, a transcriptional upregulation of VEGF was seen by an average VEGF/ $\beta 2$-M ratio that was at least 2.4 times higher than in controls.

The median (range) $\mathrm{HO}-1 / \beta 2 \mathrm{M}$ ratio in the remote area was $3.1(1.1-8.5)$ in the control group and $5.4(4.1-8.3)$ in the PICSO group. In the PICSO group, a statistically significant $(P<.001)$ upregulation of HO-1 transcription of about 6-fold was induced in the infarcted myocardium in comparison with the control group (Figure E7). In contrast to the expression pattern of VEGF, no significantly different change of HO-1 transcription was observed in the border areas of differently treated animals, thus indicating the involvement of a quantitative difference in the mechanosensitive signaling mechanism.

Electrophoretic mobility shift assays of extracts isolated from myocardial specimens showed no differences in binding of HIF- $1 \alpha$ to the consensus sequence between PICSOtreated animals versus controls (Figure E8).

To determine the interrelationship between transient systolic pulsation of the coronary sinus and the transcriptional upregulation of HO-1 and VEGF, we performed a correlation analysis. As shown in Table E2, a positive and statistically significant correlation between both mean and developed peak coronary sinus pressure and the $\mathrm{HO}-1$ and $V E G F$ gene transcription was found. These findings strongly suggest that mechanical strain and stretch produced by the pulsatile action of the reflowing blood in the deprived venous vasculature trigger the activation of a genomic program within the myocardium. 


\section{Discussion}

\section{Major Findings}

PICSO effects and their comparison with clinically applied methods of myocardial protection are well established. ${ }^{15,16}$ As reported in the literature on mechanotransduction, the intentional activation of venous endothelium by PICSO induced angiogenic cascades. ${ }^{8-10,17}$ As anticipated, the effect of the pressure increase in the coronary venous circulation influenced the local expression of our exemplary signaling molecules HO-1 and VEGF in porcine myocardium, whereas the HIF pathway remained unchanged. This might not be restricted to the endothelium inasmuch as interstitial fluid flow from the bloodstream to the media transmits shear stress to the smooth muscle cells. ${ }^{17}$

HO-1 catabolizes heme into three products: carbon monoxide, biliverdin (which is rapidly converted to bilirubin), and free iron (which leads to the induction of ferritin, an iron-binding protein). $\mathrm{HO}-1$ serves as a protective gene by virtue of the anti-inflammatory, antiapoptotic and antiproliferative actions of one or more of these three products. ${ }^{18,19}$ Lakkisto and associates ${ }^{20}$ demonstrated that the expression of HO-1 mRNA is induced after myocardial infarction in rats mainly in the infarct border area. This was accompanied by increased HO-1 protein in the cardiomyocytes of the peri-infarct border areas and in the vascular structures throughout the myocardium. These observations indicate a function for HO-1 in maintaining vascular homeostasis and perfusion in the surrounding areas of infarction by carbon monoxide-induced vasodilatation and inhibition of platelet aggregation. The importance of HO-1 as a defense mechanism against ischemia/reperfusion injury is supported by the findings of Yet and associates, ${ }^{21}$ who showed increased apoptosis around the infarcted area in hearts of HO-1 knockout mice. Besides its direct effects, HO-1 appears to be required for the action and induction of several other molecules, for example, VEGF. Accordingly, it was shown that activation or overexpression of HO-1 augments the synthesis of VEGF in vascular smooth muscle cells and in microvascular endothelium. ${ }^{22-25} \mathrm{~A}$ recent study demonstrated that transfer of HO-1 cDNA into endothelial cells results in a 2 -fold increase in blood vessel formation. ${ }^{25} \mathrm{In}$ duction of HO-1 or supplementation of cells with carbon monoxide-releasing compounds results in increased formation of capillaries. ${ }^{18,22-24}$ These observations indicate that HO-1 is, in addition to its antiapoptotic and anti-inflammatory properties, a proangiogenic factor. The angiogenic response of endothelial cells to overexpression of the $\mathrm{HO}-1$ gene provides direct evidence that the induction of HO-1 after injury represents an important tissue adaptive mechanism for moderating the severity of cell damage produced in inflammatory reaction sites of ischemia. Furthermore, it was shown that HO-1 promotes VEGF-driven angiogenesis that facilitates tissue repair. ${ }^{17}$ Wagner and associates ${ }^{26}$ reported that hemodynamic forces induce the expression of HO-1 and carbon monoxide production in cultured vascular smooth muscle cells, whereas nitric oxide synthase expression and nitric oxide production were not influenced. On the other hand, Bussolati and coworkers ${ }^{27}$ provided evidence that VEGF induces prolonged HO-1 expression and activity in human endothelial cells.

Upregulation of $V E G F$ and $H O-1$ gene expression in hypoxic and ischemic tissues is a widely known reaction and compensatory effect. Significant improvements in capillary density have also been observed with ischemic preconditioning, but clinical practicability in acute coronary syndromes is limited. In addition, numerous studies showed molecular changes of mechanotransduction on the activation of endothelium as well as subsequent cascades, supposedly leading toward recovery of jeopardized myocardium. Again, most of the methods inducing mechanical power are difficult to apply clinically or are even impractical. ${ }^{1}$ In the present study, we report on a clinically feasible method, able to strongly upregulate $\mathrm{HO}-1$ gene expression in the infarct area of PICSO-treated animals, together with an enhanced transcription of VEGF in infarct and border areas as observed in untreated controls. The statistically significant correlation $(P<.01)$ of coronary sinus pressure and the expression of HO-1 and VEGF mRNA indicates that the transient occlusion of the coronary sinus with its subsequent elevation of venous pressure and pressurization of the venous microcirculation is, in addition to ischemia, an important stimulus for the manifold expression. With respect to the short duration of the experiments, an enhanced protein expression might not have been detectable and is a limitation of the study. The tendency in treated animals to conserve global hemodynamics and ventricular dimensions, a reduced infarct size, and a preservation of functional parameters in PICSO-treated animals are positive reactions to this method, although the mechanism might not only be related to the angiogenic pulses observed, but rather caused by a vasodilatatory effect of these molecules and an enhancement of collateral circulation, especially in border zones. $^{22}$

Sharma, Das, and Verdouw ${ }^{28}$ examined the expression of HO-1 in the heart during occlusion and subsequent reperfusion of the LAD in pigs. In the distribution territory of the LAD, the expression of HO-1 was significantly increased (1.9-fold) compared with that of the area perfused by the left circumflex coronary artery (control). The authors suggested that the myocardial adaptive response to ischemia involves upregulation of HO-1, indicating that this enzyme may participate in regulating the vascular tone via carbon monoxide and thereby contribute to an important defense mechanism in the heart. In our study we found a 4-fold upregulated expression of HO-1 in the infarct area of PICSO-treated animals, which indicates that the temporary 
occlusion of the coronary sinus is an additional stimulus for HO-1 transcription. As a consequence, a carbon monoxidemediated vasodilatory response and improved perfusion in the ischemic area from its borders should occur. Besides the upregulation of HO-1 mRNA in the area of myocardial infarction, we found that PICSO further enhances the transcription of VEGF in both infarct and border areas and exceeds the known increase induced by hypoxia by at least 2.4-fold. VEGF is an endothelium-specific cell mitogen linked with vessel development during embryonic development. VEGF is produced in tissues acquiring new capillary networks; in adult tissues, expression of VEGF is increased by hypoxia and tissue ischemia. In myocardial infarction, induction of VEGF may serve as a signal for physiologically necessary compensatory angiogenesis, which may ameliorate the effects of hypertrophy and remodeling. Recent reports on the acute induction of collateralization of ischemic myocardium by PICSO seem to corroborate the notion that vasodilatory effects might be involved. ${ }^{29}$

In a rat myocardial infarction model of angiogenesis, $\mathrm{Li}$ and colleagues ${ }^{30}$ observed increased VEGF expression in the areas of the left ventricle remote from the infarct area, as well as in the right ventricular myocardium, and postulated that a nonischemic or nonhypoxic stimulus was responsible for the observed induction of VEGF expression. Intermittent coronary venous pressure increase seems to act accordingly via the pressurization of the endothelial cytoskeleton and most probably via pericytes.

Venous endothelial cells are subjected to mechanical forces, including shear stress, pressure, and stretch, which alter their functional and structural properties. ${ }^{31}$ Compared with shear stress, pulsatile stress on endothelial cells produces a "two order of magnitude" effectiveness. So far, the effects of mechanical forces on the endothelium have not been discriminated between arterial and venous microcirculation. It can be expected that mechanotransduction of venous endothelium will be the target of future research because of its unique properties in immune defense and regeneration. Zheng and associates ${ }^{32}$ demonstrated that VEGF transcription is upregulated in coronary microvascular endothelial cells, not only via paracrine signals from stretched cardiac myocytes but also via an autocrine signaling from stretched endothelial cells. Our intervention mainly increases pressure in the venous vasculature, refilling the erectile function of the transmural veins and reopening collateral microcirculation in the border zones by the squeezing action of the remaining normally functioning myocardium, but also via a molecular cascade that induces vasodilatation and subsequent acute collateralization. ${ }^{29}$

\section{A Paradigm Change in Coronary Sinus Interventions}

Our findings suggest that a biomechanical intervention using the abrupt change in venous flow and pressurization of the backed-up blood cushion in the venous capacitance induces a genomic program that ameliorates the effects of myocardial injury during coronary occlusion by the upregulation of anti-inflammatory, antioxidative, vasodilatory, and proangiogenic processes, thus enhancing the salvaging process, and by inducing long-lasting effects on the structural and functional integrity of the former ischemic zone. This might be the molecular starting point leading to the structural changes of increased vessel density seen in early experimental retroperfusion studies. This represents a paradigm change in the perception of retroperfusion techniques and a starting point in the awareness of beneficial effects and applications of this intervention. On the one hand, this may represent a promising therapeutic strategy for neovascularization in ischemic heart diseases free of ethical concerns, immune reaction, or the risk of malignancy. On the other hand, it warrants new clinical trials, such as extending coronary sinus interventions in a PISCO mode after global arrest in the early reperfusion mode or as analog to ischemic preconditioning in off-pump surgery. Komamura, Mishima, and Kodama ${ }^{1}$ presented a series of patients with acute myocardial infarction and lysis therapy and found that 40 minutes of intermittent coronary sinus occlusion had a longlasting effect, significantly reducing the incidence of major adverse cardiac events in the treated group over a period of 4 years. In contrast to autotransplantation of cells into an unfamiliar environment, PICSO induces a local environmental change resulting in activation and many-fold increase of proangiogenic gene expression within the heart as compared with controls. It can be speculated that this genetic reprogramming according to environmental changes may recruit stem cells, represents a form of "regional embryonalization," and may induce quasidevelopmental maturations of jeopardized myocardium. The long-term effects observed clinically and the structural changes seen by Hahn and $\mathrm{Kim}^{6}$ are in agreement with the starting point of molecular changes, related to the pressure changes in the venous circulation during PICSO presented in this study.

Transient elevation of coronary venous pressure and PICSO has been proven valid to salvage ischemic myocardium, to induce washout of toxic metabolites, and to improve regional myocardial blood flow and function. Our present findings on the release of angiogenic pulses suggest that, together with established beneficial results, there is a second window of myocardial protection leading to a longterm change. Recent reports from Nishida and coworkers ${ }^{2}$ on the use of mechanical force show equivalent effects on new vessel development through the application of shock waves on ischemic myocardium.

There are currently patients recruited for the Cellwave Study (Combined Extracorporal Shock Wave Therapy and Intracoronary Cell Therapy in Chronic Ischemic Myocardium) to evaluate the application of shock waves as a 
method to help to improve the homing of stem cells in chronically ischemic areas. ${ }^{33}$ In analogy, it can be speculated that periodic activation of venous endothelium through PICSO induces long-lasting effects on the healing process and eventually the functional recovery of the heart, recapitulating the environmental processes during organogenesis.

\section{Conclusions}

Our observation sheds light on the historic findings closing the cycle of scientific perception and opening a new, clinically feasible track to study the biological cause of applying controlled stresses to venous endothelium. These data provide strong evidence in support of the concept that local changes in endothelial tissue structure and mechanics resulting from hemodynamic force contribute significantly to developmental as well as regenerative response and might represent the notion "Beck and back" presented by Dwight E. Harken, ${ }^{34}$ who as a surviving contemporary of Beck ended this lecture quoting a phrase accredited to Sir Isaac Newton: "We see so far because we stand on the shoulders of giants."

This report is dedicated to our mentors and teachers in cardiothoracic surgery and experimental medicine, Prof Dr E. Wolner and Prof Dr Udo Losert, on the occasion of their 65th birthdays.

\section{References}

1. Komamura K, Mishima M, Kodama K. Preliminary clinical experience with intermittent coronary sinus occlusion in combination with thrombolytic therapy in acute myocardial infarction. Jpn Circ J. 1989; 53:1152-63.

2. Nishida T, Shimokawa H, Oi K, Tatewaki H, Uwatoku T, Abe K, et al. Extracorporeal cardiac shock wave therapy markedly ameliorates ischemia-induced myocardial dysfunction in pigs in vivo. Circulation. 2004;110:3055-61.

3. Kanamori T, Watanabe G, Yasuda T, Nagamine H, Kamiya H, Koshida Y. Hybrid surgical angiogenesis omentopexy can enhance myocardial angiogenesis induced by cell therapy. Ann Thorac Surg. 2006; 81:160-8.

4. O Shaughnessy L. Experimental methods of providing collateral circulation to the heart. Br J Surg. 1936;23:665-70.

5. Syeda B, Schukro C, Heinze G, Modaressi K, Glogar D, Maurer G, et al. The salvage potential of coronary sinus interventions: metaanalysis and pathophysiologic consequences. J Thorac Cardiovasc Surg. 2004;127:1703-12.

6. Hahn RS, Kim M. Revascularization of the heart. Histologic changes after arterialization of the coronary sinus. Circulation. 1952;5:810-5.

7. Pearson H. The heart of the matter. Nat Med. 2004;10:445-6.

8. Helmke BP. Molecular control of cytoskeletal mechanics by hemodynamic forces. Physiology (Bethesda). 2005;20:43-53.

9. Li S, Huang NF, Hsu S. Mechanotransduction in endothelial cell migration. J Cell Biochem. 2005;96:1110-26.

10. Heil M, Schaper W. Influence of mechanical, cellular, and molecular factors on collateral artery growth (arteriogenesis). Circ Res. 2004;95: 449-58.

11. Li J, Hampton T, Morgan JP, Simons M. Stretch-induced VEGF expression in the heart. J Clin Invest. 1997;100:18-24.

12. Seko Y, Seko Y, Takahashi N, Shibuya M, Yazaki Y. Pulsatile stretch stimulates vascular endothelial growth factor (VEGF) secretion by cultured rat cardiac myocytes. Biochem Biophys Res Commun. 1999; 254:462-5.

13. Matthews JNS, Altman DG, Campbell MJ, Royston P. Analysis of serial measurements in medical research. Br Med J. 1990;300:230-5.
14. Vickers AJ, Altman DG. Statistics notes: analysing controlled trials with baseline and follow up measurements. Br Med J. 2001;323: $1123-4$.

15. Lazar HL. Advantages of pressure-controlled intermittent coronary sinus occlusion over left ventricle-powered coronary sinus retroperfusion. Ann Thorac Surg. 2001;71:402.

16. Lazar H. Coronary sinus retroperfusion: can forward progress still be achieved by using a backward technique? J Thorac Cardiovasc Surg. 2004;127:1549-52.

17. Dulak J, Motterlini R, Huk I, Pachinger O, Weidinger F, Jozkowicz A. Carbon monoxide and iron, by-products of heme oxygenase, modulate vascular endothelial growth factor synthesis in vascular smooth muscle cells. In: Abraham NG, editor. Physiology and pathology of heme oxygenase. New York: Kluwer; 2002. p. 97-107.

18. Otterbein LE, Soares MP, Yamashita K, Bach FH. Heme oxygenase-1: unleashing the protective properties of heme. Trends Immunol. 2003; 24:449-55.

19. Otterbein LE, Bach FH, Alam J, Soares M, Lu HT, Wysk M, et al. Carbon monoxide has anti-inflammatory effects involving the mitogenactivated protein kinase pathway. Nat Med. 2000;6:422-8.

20. Lakkisto P, Palojoki E, Bäcklund T, Saraste A, Tikkanen I, VoipioPulkki LM, et al. Expression of heme oxygenase-1 in response to myocardial infarction in rats. J Mol Cell Cardiol. 2002;34:1357-65.

21. Yet SF, Perrella MA, Layne MD, Hsieh CM, Maemura K, Kobzik L, et al. Hypoxia induces severe right ventricular dilatation and infarction in heme oxygenase-1 null mice. J Clin Invest. 1999;103:R23-9.

22. Józkowicz A, Huk I, Nigisch A, Weigel G, Dietrich W, Motterlini R, et al. Heme oxygenase and angiogenic activity of endothelial cells: stimulation by carbon monoxide, inhibition by tin protoporphyrin-IX. Antioxid Redox Signal. 2003;5:155-62.

23. Dulak J, Józkowicz A, Foresti R, Kasza A, Frick M, Huk I, et al. Heme oxygenase activity modulates vascular endothelial growth factor synthesis in vascular smooth muscle cells. Antioxid Redox Signal. 2002; 4:229-38.

24. Józkowicz A, Huk I, Nigisch A, Weigel G, Weidinger F, Dulak J. Effect of prostaglandin- $\mathrm{J}_{2}$ on VEGF synthesis depends on the induction of heme oxygenase-1. Anioxid Redox Signal. 2002;4:577-85.

25. Deramaudt BM, Braunstein S, Remy P, Abraham NG. Gene transfer of human heme oxygenase into coronary endothelial cells potentially promotes angiogenesis. J Cell Biochem. 1998;68:121-7.

26. Wagner CT, Durante W, Christodoulides N, Hellums JD, Schafer AI. Hemodynamic forces induce the expression of heme oxygenase in cultured vascular smooth muscle cells. J Clin Invest. 1997;100: 589-96.

27. Bussolati B, Ahmed A, Pemberton H, Landis RC, Di Carlo F, Haskard $\mathrm{DO}$, et al. Bifunctional role for VEGF-induced heme oxygenase-1 in vivo: induction of angiogenesis and inhibition of leukocytic infiltration. Blood. 2004;103:761-6.

28. Sharma HS, Das DK, Verdouw PD. Enhanced expression and localization of heme oxygenase-1 during recovery phase of porcine stunned myocardium. Mol Cell Biochem. 1999;196:133-9.

29. Mohl W, Kajgana I, Bergmeister, H, Rattay F. Intermittent pressure elevation of the coronary venous system as a method to protect ischemic myocardium. Interact Cardiovasc Thorac Surg. 2005; 4:66-9.

30. Li J, Brown LF, Hibberd MG, Grossman JD, Morgan JP, Simons M. VEGF, flk-1, and flt-1 expression in a rat myocardial infarction model of angiogenesis. Am J Physiol. 1996;270:H1803-11.

31. Davies PF, Tripathi SC. Mechanical stress mechanisms and the cell. An endothelial paradigm. Circ Res. 1993;72:239-45.

32. Zheng W, Brown MD, Brock TA, Bjercke RJ, Tomanek RJ. Bradycardia-induced coronary angiogenesis is dependent on vascular endothelial growth factor. Circ Res. 1999;85:192-8.

33. Cellwave Study: Combined extracorporal shock wave therapy and intracoronary cell therapy in chronic ischemic myocardium.www. clinicaltrials.gov_ClinicalTrials.gov Identifier: NCT00326989; 2006.

34. Harken DE. Beck and back. In: Mohl W, Faxon D, Wolner E, editors. The Clinics of CSI. Reprinted in the Coronary Sinus Library, 2003. Society of Coronary Sinus Interventions 2003. Vol 4. p. 3-12. 


\section{*Experimental Design}

The studies were carried out on 12 female pigs (Edelschwein, $30-35 \mathrm{~kg}$ ). After premedication with $1.86 \mathrm{mg} / \mathrm{kg}$ acepromacine (Vanastress) plus $15 \mathrm{mg} / \mathrm{kg}$ ketamine (Ketavet), anesthesia was initiated with thiopentone sodium (Thiopental, $10 \mathrm{mg} / \mathrm{kg}$ ), piritramid (Dipidolor, $15 \mathrm{mg}$ ), and pancuronium bromide (Pavulon, 4 $\mathrm{mg}$ ). After endotracheal intubation, anesthesia was maintained with $100 \mu \mathrm{g} \cdot \mathrm{kg}^{-1} \cdot \mathrm{h}^{-1}$ piritramid plus $6.6 \mu \mathrm{g} \cdot \mathrm{kg}^{-1} \cdot \mathrm{h}^{-1}$ pancuronium bromide in Ringer's solution and propofol (Diprivan) $6 \mathrm{mg} \cdot \mathrm{kg}^{-1} \cdot \mathrm{h}^{-1}$. The animals were ventilated with volume-controlled oxygen and room air according to blood gas values.

\section{${ }^{\dagger}$ Echocardiography}

Two-dimensional and two-dimensionally guided M-mode echocardiograms were performed on a subset of 2 controls and 4 PICSO-treated animals with a 5-MHz transducer at baseline, 30 minutes after induction of myocardial infarction, 30 minutes after PICSO (at 60 minutes after myocardial infarction), and at 120 and 180 minutes after myocardial infarction.

${ }^{\ddagger}$ The sequences of the used primers are as follows: HO-1 Forward, 5'-CCT CTC GCT GGA AAA AAG GA-3'; HO-1 Reverse, 5'-AGG GCA CTG GCT GCA TGT-3'; VEGF Forward, 5'-CCC ACG AAG TGG TGA AGT TCA-3'; VEGF Reverse, 5'-CCA CCA GGG TCT CGA TTG G-3'. Primers and Taq Man probes were designed according to GenBank: accession numbers X60677 and AF461807, respectively. Primers were designed to exclude the amplification of genomic DNA. FAM-labeled Taq Man probes were used to detect the polymerase chain reaction product. The probe sequences were designed to be complementary to the target sequences of the $\mathrm{HO}-1$ and $V E G F$ gene. The sequence of the HO-1 probe was as follows: 5'-TGT GGC ATC TTT CCC AAC CAA- $3^{\prime}$. The sequence of the VEGF probe was as follows: 5'-CGT CTA CCA GCG CAG CTA CTG CCG-3'. Target transcripts were normalized to the endogenous housekeeping transcript $\beta 2-$ M. Primers and FAM-labeled Taq Man probe were designed according to GenBank: accession number L13854. The sequences read as follows: $\beta 2-\mathrm{M}$ Forward primer, $5^{\prime} \mathrm{CCC}$ CCG AAG GTT CAG GTT TA-3'; Reverse, 5'GCA GTT CAG GTA ATT TGG
CTT TC-3'; Taq Man probe, 5'TCA CGC CAC CCA GCG GAA AAC- $3^{\prime}$.

\section{§DNA Binding Reaction}

The double-stranded blunt-ended HIF- $1 \alpha$ (5' GCC CTA CGT GCT GTC TCA 3') consensus oligonucleotide was customersynthesized by VBC-GENOMICS (Vienna, Austria) and end-labeled with $\gamma-{ }^{32} \mathrm{P}$ adenosine triphosphate (ICN Pharmaceuticals) using T4 polynucleotide kinase (Promega Corporation, Madison, Wis). Unincorporated nucleotides were removed by centrifugation over a Sephadex G-25 spin column (Boehringer, Mannheim, Germany). Binding reactions containing $14 \mu \mathrm{g}$ of nuclear extract, $1 \mu \mathrm{g}$ poly(dI) poly(dC), and $10^{3} \mathrm{cpm}$ of $\gamma-{ }^{32} \mathrm{P}$-labeled oligonucleotide probe were incubated for 20 minutes at room temperature. ProteinDNA complexes were resolved by nondenaturing electrophoresis on $4.5 \%(\mathrm{w} / \mathrm{v})$ polyacrylamide slab gels. Gels were initially prerun in $1 \times$ Tris-glycine-ethylenediaminetetraaceitc acid buffer for 30 minutes at $300 \mathrm{~V}$; subsequent to loading of samples, electrophoresis was maintained at $300 \mathrm{~V}$ for 30 to 40 minutes. Gels were dried under vacuum and HIF- $1 \alpha$ complexes were visualized by autoradiography.

For competition experiments, a 100 -fold $\mathrm{mol} / \mathrm{L}$ excess of unlabeled annealed oligonucleotides was added to the binding reaction mixtures before addition of the labeled probes.

The specificity of DNA binding was further confirmed by a mutant oligo with a CGT $\rightarrow$ AAA substitution in the HIF- $1 \alpha$ DNA binding motif (VBC-GENOMICS, Vienna, Austria) instead of the HIF- $1 \alpha$ consensus oligonucleotide.

ITable E1 shows a summary of the hemodynamic data measured in the control group (Table E1, $A$ ) and in the PICSO group (Table E1, B).

Averaged global hemodynamics are depicted in Table E1, $C$.

\section{E-Reference}

E1. Force TL, Folland ED, Aebischer N. Echocardiographic assessment of ventricular function. In: Marcus ML, Skorton DJ, Schelbert HR, Wolf GL, editors: Cardiac imaging: a companion to Braunwald's heart disease. Philadelphia: WB Saunders; 1991, p. 374-471. 
TABLE E1A. Hemodynamic data during normal perfusion and LAD occlusion without PICSO (control group)

\begin{tabular}{|c|c|c|c|c|c|c|c|}
\hline & \multirow[b]{2}{*}{ Normal perfusion } & \multirow[b]{2}{*}{ LAD occlusion } & \multicolumn{5}{|c|}{ Time after LAD occlusion } \\
\hline & & & $15 \mathrm{~min}$ & $30 \mathrm{~min}$ & $60 \mathrm{~min}$ & $120 \mathrm{~min}$ & $180 \mathrm{~min}$ \\
\hline HR & $97.2 \pm 18.9$ & $103.2 \pm 28.5$ & $97.4 \pm 29.5$ & $99.0 \pm 23.0$ & $99.6 \pm 20.1$ & $100.0 \pm 14.5$ & $93.8 \pm 29.2$ \\
\hline AoP sys. & $111.2 \pm 11.3$ & $106.0 \pm 11.6$ & $88.6 \pm 14.3$ & $91.0 \pm 11.7$ & $92.2 \pm 9.0$ & $87.0 \pm 11.9$ & $84.5 \pm 1.3$ \\
\hline AoP dia. & $73.2 \pm 10.8$ & $65.2 \pm 11.3$ & $60.6 \pm 13.3$ & $61.0 \pm 12.9$ & $56.8 \pm 13.9$ & $58.0 \pm 4.1$ & $46.0 \pm 8.3$ \\
\hline CSP sys. & $10.0 \pm 0.0$ & $10.0 \pm 0.0$ & $7.5 \pm 3.5$ & $7.5 \pm 3.5$ & $7.0 \pm 2.8$ & $7.0 \pm 2.8$ & $7.0 \pm 2.8$ \\
\hline CSP dia. & $4.0 \pm 1.4$ & $4.0 \pm 1.4$ & $3.0 \pm 0.00$ & $3.0 \pm 0.0$ & $4.0 \pm 1.4$ & $4.0 \pm 1.4$ & $4.0 \pm 1.4$ \\
\hline
\end{tabular}

$L A D$, Left anterior descending coronary artery; PICSO, pressure-controlled intermittent coronary sinus occlusion; $H R$, heart rate (beats/min); $A o P$, aortic blood pressure (mm Hg); CSP, coronary sinus pressure; sys, systolic; dia, diastolic.

TABLE E1B. Hemodynamic data during normal perfusion, LAD occlusion, and PICSO

\begin{tabular}{|c|c|c|c|c|c|c|c|}
\hline & \multirow[b]{2}{*}{ Normal perfusion } & \multirow[b]{2}{*}{ LAD occlusion } & \multicolumn{5}{|c|}{ Time after LAD occlusion } \\
\hline & & & $15 \mathrm{~min}$ & $30 \mathrm{~min}$ & $60 \mathrm{~min}$ & $120 \mathrm{~min}$ & $180 \mathrm{~min}$ \\
\hline$H R$ & $105.5 \pm 9.6$ & $102.4 \pm 14.2$ & $97.5 \pm 28.7$ & $107.2 \pm 16.8$ & $113.3 \pm 14.8$ & $112.3 \pm 18.5$ & $110.8 \pm 14.0$ \\
\hline AoP sys & $113.0 \pm 6.0$ & $105.2 \pm 10.8$ & $91.2 \pm 16.8$ & $95.3 \pm 10.1$ & $96 \pm 11.0$ & $92.3 \pm 8.5$ & $955 \pm 10.0$ \\
\hline AoP dia & $77.5 \pm 7.8$ & $68.8 \pm 9.2$ & $62.5 \pm 9.2$ & $61.2 \pm 8.2$ & $55.8 \pm 108$ & $52.8 \pm 11.5$ & $58.5 \pm 8.2$ \\
\hline CSP sys & $19.8 \pm 4.0$ & $17 \pm 5.0$ & $46.3 \pm 19.9$ & $57.0 \pm 14.3$ & $54.5 \pm 16.9$ & $50.3 \pm 123$ & $49.3 \pm 141$ \\
\hline CSP dia & $10.2 \pm 5.0$ & $5.8 \pm 0.5$ & $17.7 \pm 10.4$ & $18.5 \pm 7.4$ & $18.2 \pm 7.7$ & $18 \pm 7.7$ & $17.7 \pm 8.3$ \\
\hline
\end{tabular}

$L A D$, Left anterior descending coronary artery; PICSO, pressure-controlled intermittent coronary sinus occlusion; $H R$, heart rate (beats/min); AoP, aortic blood pressure $(\mathrm{mm} \mathrm{Hg})$; CSP, coronary sinus pressure; sys, systolic; dia, diastolic.

TABLE E1C. Averaged hemodynamic data during intervention

\begin{tabular}{lccc}
\hline & PICSO & Controls & P value \\
\hline HR & $109.0 \pm 18.0$ & $97.8 \pm 20.1$ & .7 \\
AoP sys & $93.8 \pm 10.1$ & $88.4 \pm 9.5$ & .25 \\
AoP dia & $57.9 \pm 8.4$ & $56.7 \pm 6.2$ & .95 \\
\hline
\end{tabular}

PICSO, Pressure-controlled intermittent coronary sinus occlusion; $H R$, heart rate (beats/min); AoP, aortic blood pressure $(\mathrm{mm} \mathrm{Hg})$; sys, systolic; dia, diastolic.
TABLE E2. Correlation of myocardial gene expression and CSP

\begin{tabular}{lcc}
\hline & $\begin{array}{c}\text { Coefficient of } \\
\text { correlation }\end{array}$ & Significance \\
\hline VEGF-CSP peak & 0.60 & $P<.01$ \\
VEGF-CSP mean & 0.63 & $P<.01$ \\
HO-1-CSP peak & 0.38 & $P<.01$ \\
HO-1-CSP mean & 0.42 & $P<.01$
\end{tabular}

$C S P$, Coronary sinus pressure; VEGF, vascular endothelial growth factor; HO-1, heme oxygenase-1. 




Figure E1. Scatter plot of the ejection fraction of control and PICSO group data with an observation period of about $180 \mathrm{~min}$ utes. In both groups, the ejection fraction decreases depending on rinsing time (PICSO $\boldsymbol{P}<.06$; control $\boldsymbol{P}<.004$ ). The ejection fraction decrease was not statistically significant between the groups $(P=.08)$. PICSO, Pressure-controlled intermittent coronary sinus occlusion.



Figure E2. Scatter plot of the mean end-diastolic dimension (EDD) of control and PICSO group data with an observation period of about 180 minutes. The mean EDD increases in both groups depending on rinsing time, however without reaching statistical significance. The mean EDD increase was not significantly different in the groups $(P=.14)$. PICSO, Pressure-controlled intermittent coronary sinus occlusion. 




Figure E3. Scatter plot of the mean end-systolic dimension (ESD) of control and PICSO group data with an observation period of about 180 minutes. The mean ESD increases in both groups depending on rinsing time, reaching statistical significance only in the control group $(P<.03)$. The mean ESD increase was not significantly different between the groups $(P=.21)$. PICSO, Pressure-controlled intermittent coronary sinus occlusion.

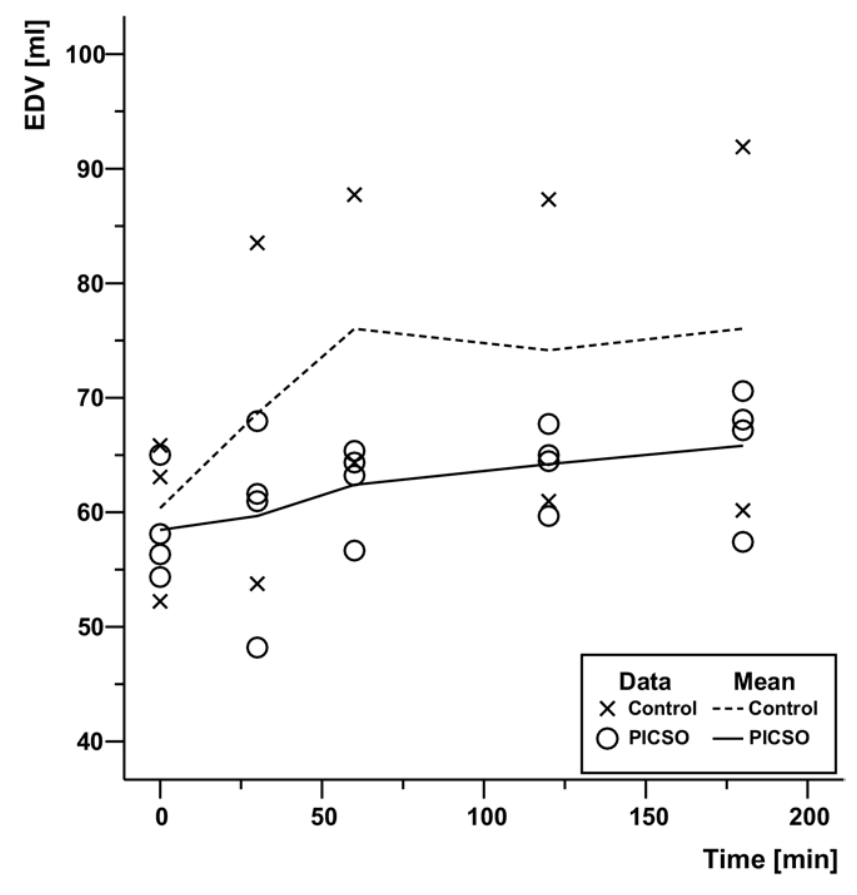

Figure E4. Scatter plot of the end-diastolic volume (EDV) of control and PICSO group data with an observation period of about 180 minutes. The EDV increases in both groups depending on rinsing time, reaching statistical significance in the PICSO group $(P<$ .03). The EDV increase was not significantly different between the groups $(P=.11)$. PICSO, Pressure-controlled intermittent coronary sinus occlusion. 




Figure E5. Scatter plot of the end-systolic volume (ESV) of control and PICSO group data with an observation period of about 180 minutes. The ESV increases in both groups depending on rinsing time, reaching statistical significance in the PICSO group $(P<$ .04). The ESV increase was not statistically significant between the groups $(P=.18)$. PICSO, Pressure-controlled intermittent coronary sinus occlusion.

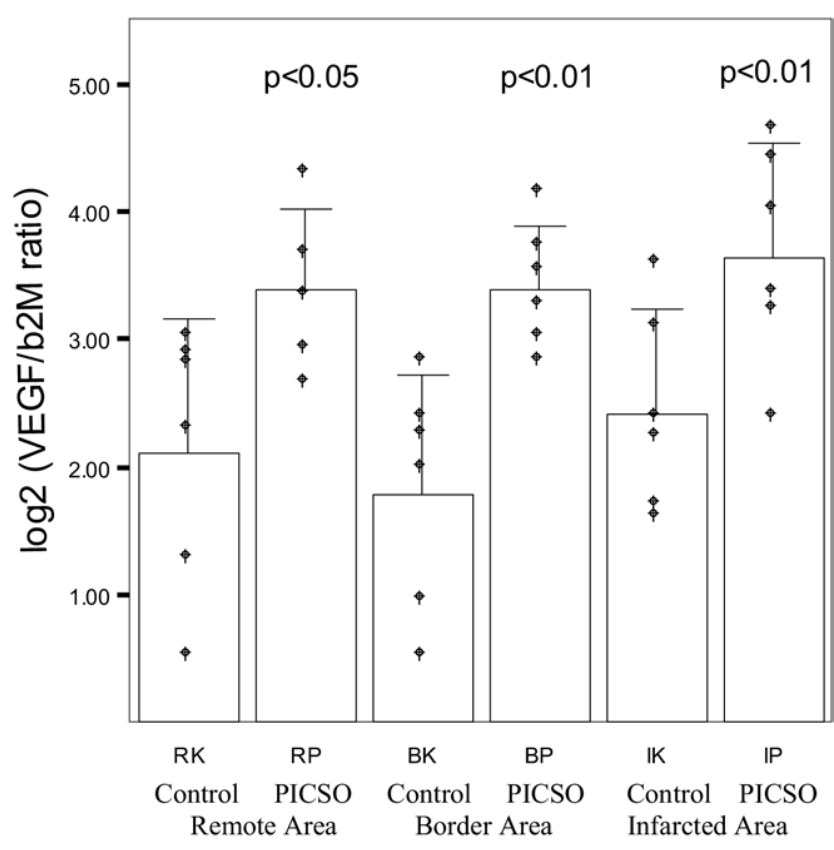

Figure E6. Expression of VEGF mRNA in different specimens of remote, border, and infarcted areas of porcine myocardium. Transient pressurization of the coronary sinus (PICSOT) induces a significant upregulation of VEGF expression compared with controls $(t)$. Student 2-sample $t$ test on log-base-2 transformed data. VEGF, Vascular endothelial growth factor; PICSO, pressure-controlled intermittent coronary sinus occlusion. 


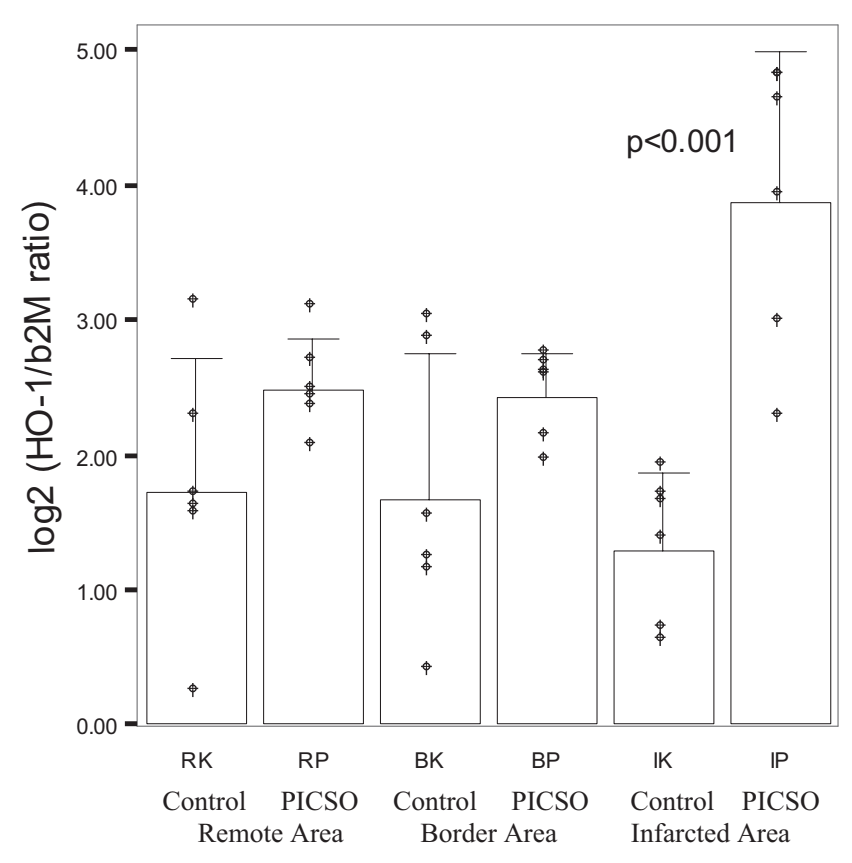

Figure E7. Expression of H0-1 mRNA in different specimens of remote, border, and infarcted areas of porcine myocardium. Transient pressurization of the coronary sinus (PICSOT) induces a significant upregulation of $\mathrm{HO}-1$ expression in the infarcted area compared with controls $(t)$. Student 2-sample t test on log-base-2 transformed data. H0-1, heme oxygenase 1; PICSO, pressurecontrolled intermittent coronary sinus occlusion.

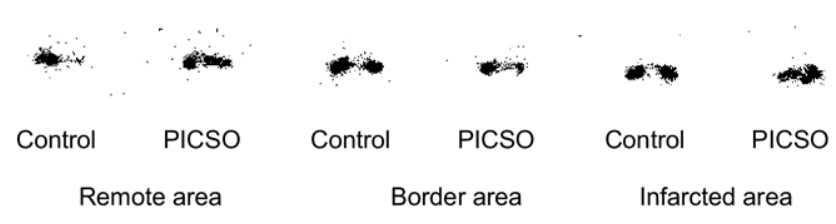

Figure E8. Representative electrophoretic mobility shift assay of tissue extracts isolated from remote, border, and infarcted areas of porcine myocardium. PICSO, Pressure-controlled intermittent coronary sinus occlusion. 